\title{
High Resolution Molecular Radiation Therapy and Tumor Imaging for the $21^{\text {st }}$ Century
}

\section{Anders Brahme*}

Department of Medical Radiation Physics, Karolinska Institute, Stockholm, Sweden

\begin{abstract}
With the lightest ions beyond protons, i.e., Helium, Lithium and Beryllium ions, highly specific Molecular Bragg peak radiation therapy of malignant tumors is possible with minimal adverse normal tissue reactions elsewhere in the body. The Bragg peak ionization density is only elevated in a few $\mathrm{mm}$ wide spot at the end of the ion range with resultant increased local apoptosis and senescence. By only placing Bragg peaks in the tumor, an increased local therapeutic effect is obtained with only low ionization density and easily repairable damage in surrounding normal tissues. A geometrical accuracy in dose delivery of about $1 \mathrm{~mm}$ is possible with these ions, and high-resolution molecular tumor imaging is then needed to accurately delineate the target volume. It is proposed that ultra-sensitivity whole body PET cameras should be built to achieve $\mathrm{mm}$ resolution in the whole target region. With about $1 \mathrm{~m}$ axial field of view an almost 50 -fold increased sensitivity and a reduced imaging time down to a few minutes should be in reach. To get sub $\mathrm{mm}$ resolution with whole body spectroscopic MR, about 15 Tesla to 20 Tesla is needed and will significantly increase the resolution with tumor specific metabolite imaging from the $10 \mathrm{~mm}$ to $15 \mathrm{~mm}$ available today. In the future, it should also be possible to achieve a resolution as high as $10 \mu \mathrm{m}$ with Stereoscopic Phase Contrast X-ray imaging, or to reduce the dose and imaging time by using 2 projections instead of 400 to get 3D images, thanks to the significantly increased contrast in each projection. When these new methods are brought into clinical use together with light ion therapy a mean tumor cure as high as $80 \%$ should be possible, and even more if the new early tumor detection and malignancy estimation methods are brought into more regular clinical use.
\end{abstract}

Keywords: Molecular radiation; Radiation therapy; Tumors; Tumor imaging

\section{Introduction}

There are a number of potential important developments in the therapeutic and imaging arenas that not yet has reached clinical application. Today most therapeutic methods have reached sub $\mathrm{mm}$ accuracy in their $3 \mathrm{D}$ dose delivery and consequently we need this degree of accuracy also in our diagnostic imaging techniques. CT and MR have already reached this geometrical accuracy but not when we require highly specific molecular tumor imaging. With MR the tumor specific metabolite imaging still has a resolution around $10 \mathrm{~nm}$ to 15 $\mathrm{mm}$ which could be brought down to the sub $\mathrm{mm}$ level by very high field strength MR units. Dual energy CT may increase the highly tumor specific imaging, not least using molecular contrast agents, but the ultimate development of 3D high resolution $\mathrm{X}$-ray imaging may require the use of stereoscopic phase contrast flash X-rays with potential sub $\mathrm{mm}$ to $\mu \mathrm{m}$ resolution in live humans. PET and PET-CT have reached about $4 \mathrm{~mm}$ tumor specific resolution in whole body imaging, and ultra-sensitive PET units with a very large field of view may improve this significantly down to about $1 \mathrm{~mm}$. This would make positron diffusion unfolding feasible to improve the geometric resolution. With all these diagnostic developments, there is a high interest to also develop molecular radiation therapy to sub $\mathrm{mm}$ accuracy using the highly specific apoptotic cell kill and senescence induction available in the few $\mathrm{mm}$ size.

Bragg peak of Lithium ions such beams would everywhere else are characterized by a low intensity and easily repairable sparsely ionizing interactions. This potentially very effective kind of molecular radiation therapy is probably the ultimate modality for highly curative local cancer treatments. Interestingly, all these developments are at first sight costly, but due to faster diagnostic methods and fewer treatment fractions needed, only 10 to 15 treatments instead of 30, they are still cost effective enough to significantly reduce the total cost for high quality curative cancer treatments.

\section{The Molecular Mechanisms of Radiation Therapy}

To optimize equipment design it is important to have a clear-cut view of the molecular function and underlying principles of radiation therapy. It was quite early understood that the optimal use of radiation cure generally required multiple daily dose fractions so the normal tissues had a possibility to repair their damage from one day to the next, whereas the tumor had more difficult to do so. Today we know that cancers often develop due to the genetic instability of some cells in the human body. This may result in mutant cell lines that proliferate more rapidly and they may even mutate further due to the genomic instability to produce potential invasive cancer cells.

Interestingly, these cancer cells evade the normal homeostatic control of the body tissues since some of their DNA damage surveillance genes like p53 and repair genes like ATM, DNApk and many others, often are defective in most, if not all, tumors (Figure 1) [1-3].

Therefore, DNA damaging agents are quite effective as a therapeutic modality since they hit the genetic instability that is the Achilles heal of most tumors. Many tumors seem to have quite effective DNA damage repair systems, but the fidelity of their repair is low and the normal cell cycle blocks required for high repair quality are often missing. A tumor cell may therefore, instead of stopping the cell cycle to do high fidelity

*Corresponding author: Anders Brahme, Department of Medical Radiation Physics, Karolinska Institute, Box 260, SE-171 76 Stockholm, Sweden, Tel: 46706155534; E-mail: anders.brahme@ki.se

Received September 22, 2016; Accepted October 13, 2016; Published October 30, 2016

Citation: Brahme A (2016) High Resolution Molecular Radiation Therapy and Tumor Imaging for the 21 $1^{\text {st }}$ Century. J Nucl Med Radiat Ther 7: 311. doi: 10.4172/2155-9619.1000311

Copyright: (C) 2016 Brahme A. This is an open-access article distributed under the terms of the Creative Commons Attribution License, which permits unrestricted use, distribution, and reproduction in any medium, provided the original author and source are credited. 


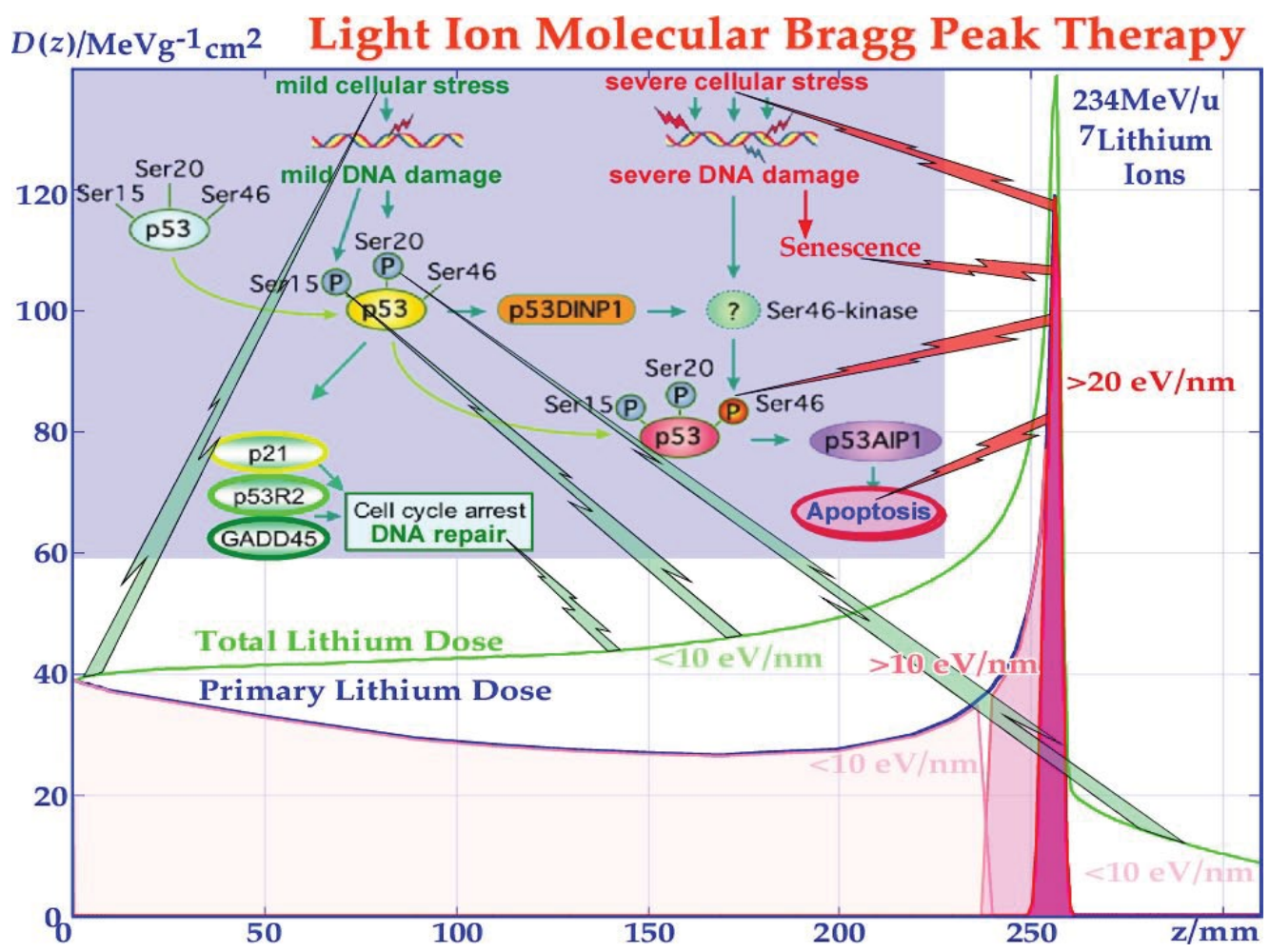

Figure 1: The DNA damage in the cell is recognized by proteins that signal their results by phosphorylation on the p53 gene product ("the guardian of the genome"). Low ionization density damage generally phosphorylates the serine 15 and 20 sites on p53 which leads to a cell cycle block and initiation of DNA repair by $\mathrm{p} 21$ and GADD45. With lithium ions, this mechanism dominates in normal tissues in front of and behind the tumor [2]. At the Bragg peak both the ionization density and dose are high, which leads to more severe unrepairable DNA damage which commonly phosphorylates the serine 46 site on p53 and thereby triggers an apoptotic response, eliminating tumor cells with severe DNA damage [1].

repair and then continue dividing, directly continue DNA synthesis and incorporate some damaged DNA in their genome.

This is probably the mechanism that makes tumor cells more sensitive to radiation damage than most normal tissues with welldeveloped cell cycle blocks and highly accurate DNA repair systems like Homologous Recombination (HR) and Non Homologous End Joining (NHEJ) as shown in Figures 1 and 2. The use of daily fractionated radiation therapy is therefore a powerful method to stepwise increase the damage to tumor cell DNA at the same time as normal tissues can repair most of their damage over night with high fidelity to be ready for the next fraction. The tumor cells on the other hand will accumulate more and more damage to their DNA for each treatment fraction. The first fractions may not be as lethal but as more and more defect DNA is accumulated in the tumor cells they will soon receive a wide panorama of damage including severe effects on genes that are essential for survival. Therefore, it will be more and more difficult for the tumor cells to survive and divide, which finally may lead to cell death for example by mitotic catastrophe. The normal tissues with their high fidelity repair systems can instead repair as many as $99 \%$ of their double strand breaks (Figure 2) [4] at the optimal 2 Gy level to be ready for the next fraction over night [5]!

Furthermore, as the dose to normal tissues with modern radiation modalities and irradiation technique can be kept rather low, the complication-free cure can be very high, for example using light ions and intensity modulated photons. One may then think that the highest dose possible should be delivered to the tumor, but this may not always be desirable, for example if the tumor is growing in an organ with essential function for patient survival. The beauty of radiation therapy is that the dose-delivery often can be chosen such that stem-cells of the normal tissue are tolerating the irradiation, whereas the tumor cells due to their genomic instability with disturbed cell cycle check points e.g. due to mutant p53, may be effectively eradicated as seen in Figure 3. It is seen that partly destructed bony structure of this bone and soft tissue sarcoma is almost fully recovered a few years after carbon ion therapy. In such cases the dose delivery should be as mild as possible to ensure normal tissue function, but high and frequent enough to eradicate all clonogenic tumor cells with good cosmetic results in normal tissues like skin and bone.

\section{Molecular Light Ion Therapy for the $21^{\text {st }}$ Century}

From the above discussion, the ultimate modality for radiation therapy is probably Light ions in the range from Helium $(Z=2)$ to Carbon $(Z=6)$ as shown in Figure 4. Helium is interesting for its potential sharp penumbra ( $1 / 2$ of that of photons and protons) and fairly low ionization density, whereas Carbon ions have a highly elevated ionization density in the last five $\mathrm{cm}$ of their range.

So, Helium ions are probably ideal for small rather well oxygenated tumors, whereas Boron $(\mathrm{Z}=5)$ and Carbon ions will be needed for large hypoxic tumors requiring an increased ionization density. For small to medium sized tumors Lithium $(\mathrm{Z}=3)$ and Beryllium $(\mathrm{Z}=4)$ ions may often be optimal since they will deliver a low ionization density to all normal tissues outside of the tumor volume, whereas only their sharp Bragg peaks ( $2 \mathrm{~mm}$ to $5 \mathrm{~mm}$ ) have a high ionization density which will 


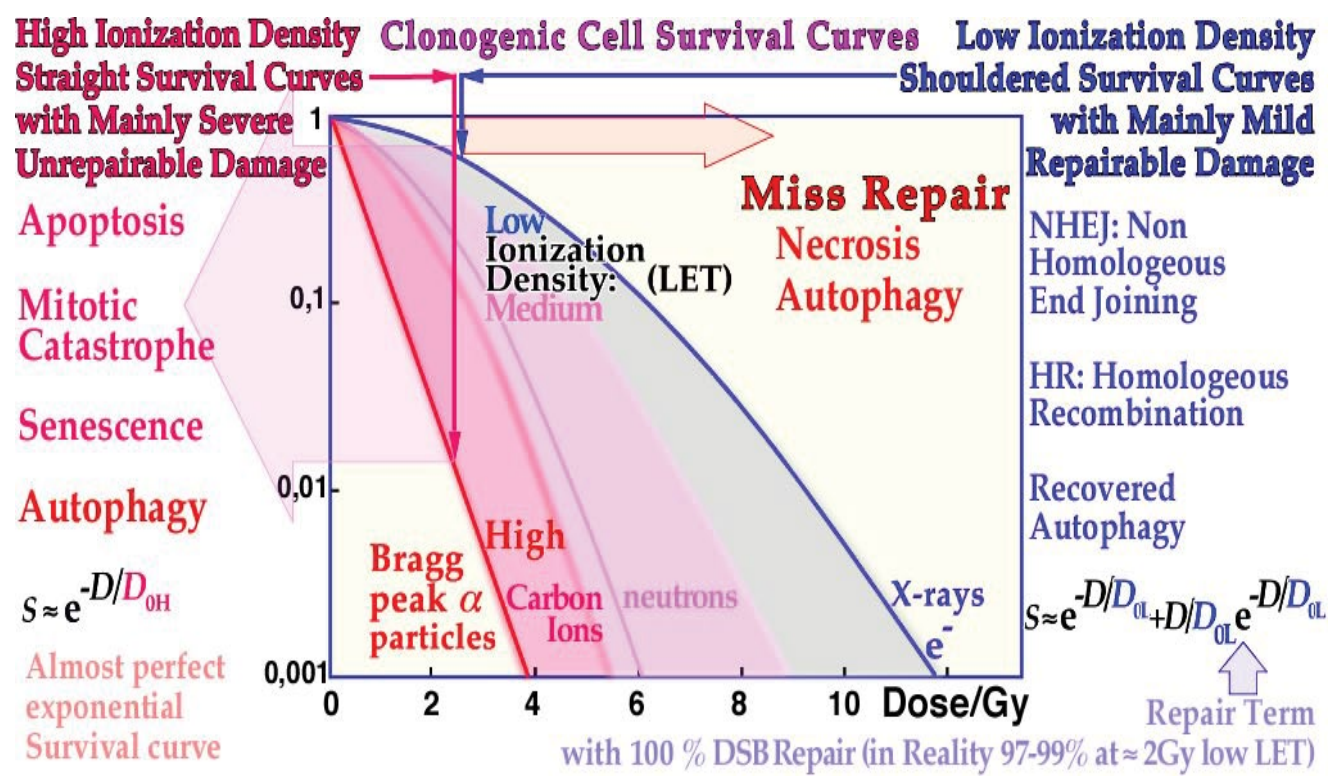

Figure 2: The shape of the clonogenic cell survival curve depends on the type of cellular damage and its reparability by the key repair pathways; NonHomologous End Joining (NHEJ) and Homologous Recombination (HR). NHEJ is generally the first process making sure that free DNA ends are joined together as fast as possible to minimize disrepair. This process is error prone and HR is capable to proofread the repair in the S2-M phase of the cell cycle based on the homologous chromosome from the other parent.

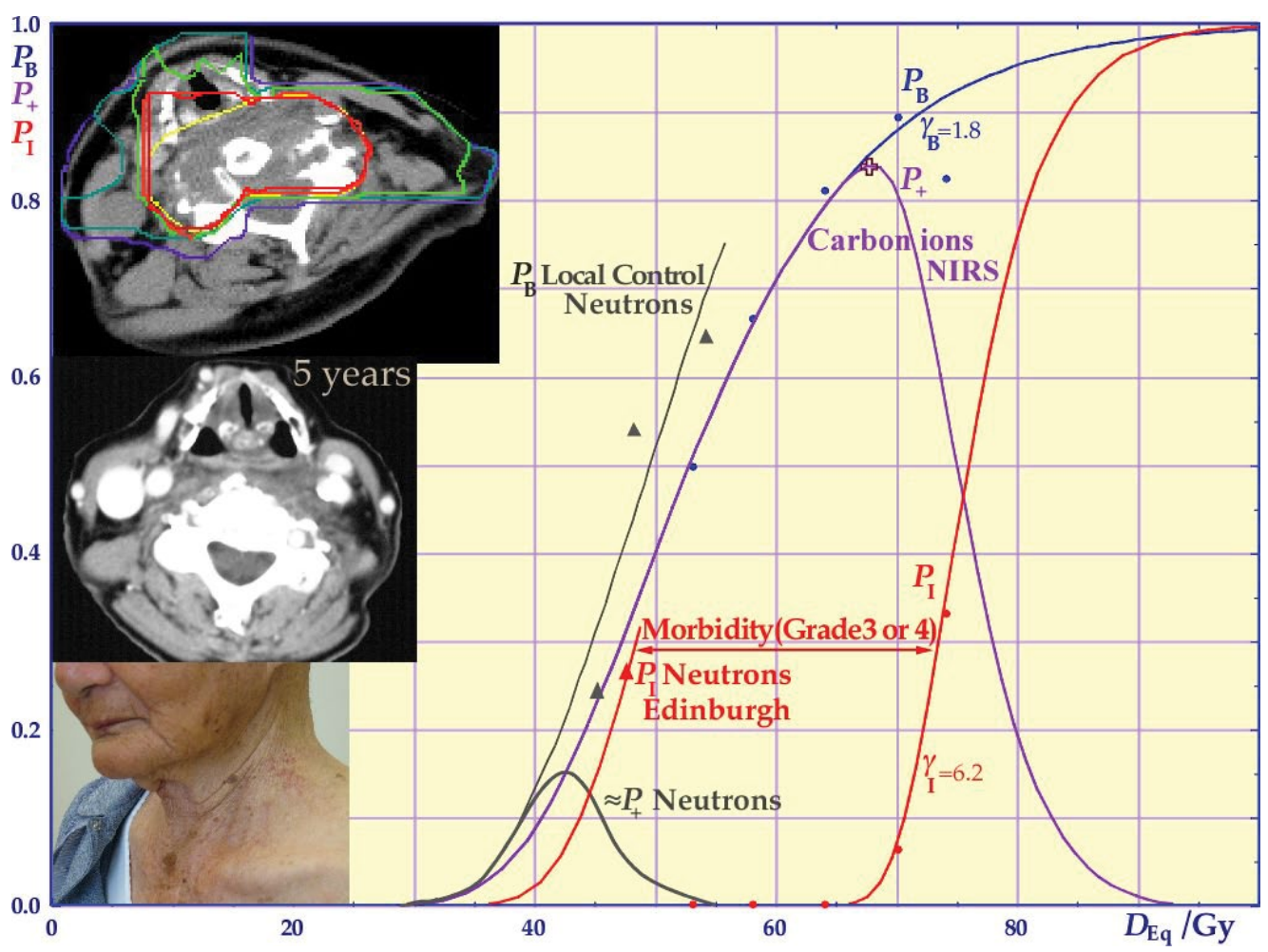

Figure 3: Bone and soft tissue sarcoma are very effectively treated by carbon ions similar to neutrons $\left(\mathrm{P}_{\mathrm{B}}\right)$. However, the normal tissue morbidity is much lower with ions since the LET in normal tissue is much lower for carbon ions than for neutrons, resulting in a significantly increased complication-free cure $\approx 85 \%$ for carbon ions and about $15 \%$ for neutrons). The very good cosmetic results and healing of the bony structures are seen in the lower and middle left inserts (Clinical data: NIRS, Chiba, Dose response modeling: Karolinska).

eradicate hypoxic tumor cells more effectively. At the same time, normal tissues are well protected from the more complex damage generated by the high ionization density that often is lethal both to the normal tissues and to the tumor. Carbon ions, on the other hand, have a high ionization 
density already $4 \mathrm{~cm}$ to $5 \mathrm{~cm}$ in front of the Bragg peak and some $10 \mathrm{~cm}$ behind it. So, when this area falls into normal tissues significant damage may occur even if the dose is fairly low (Figure 4). With Boron ions, this effect is reduced, so they may be the optimal ions for medium to large tumors that do not suffer from severe hypoxia requiring the somewhat higher ionization density of Carbon ions. Ions beyond Carbon are generally less interesting as a large part of the clinical advantage of a high tumor cell kill and minimal normal tissue damage is unfortunately lost. This is so since sensitive normal tissues in front of and behind the tumor may be severely affected due to the high ionization density both in front of and behind the Bragg peak. The lightest ions beyond protons, mainly Helium, Lithium and Beryllium, have a unique property of a high ionization density only in the region of the Bragg peak (Figures 1 and 4) [6]. These ions are therefore associated with an almost surgical property delivering cell kill with a high ionization density only around the Bragg peak. Interestingly, a large part of this cell kill is delivered by inducing apoptosis or programmed cell death (Figure 1).

Apoptosis is nature's own way to eliminate unwanted cells during the development of practically all organs and is therefore not generally associated with an inflammatory response accompanying the more common necrotic type of cell kill. Furthermore, these light ions also have an increased induction of senescence, that is, permanent cell cycle arrest. Senescence is therefore probably the most desirable endpoint of all cancer therapies as the tumor cells lose their reproductive ability and are then slowly disappearing depending on the remaining cellular lifetime. As seen in Figure 5 the tumor was reduced to about half the size or $10 \%$ to $15 \%$ of the initial volume six years after the treatment. Due to the fact that more ions per unit dose and cell kill are needed at medium to low ionization density, more effective apoptotic and senescent response is obtained at an ionization density of around 20 $\mathrm{eV} / \mathrm{nm}$ to $40 \mathrm{eV} / \mathrm{nm}$ as shown theoretically and experimentally [7]. Interestingly, Helium, Lithium and Beryllium combines a high local apoptotic and senescent tumor cell inactivation only a few $\mathrm{mm}$ around their Bragg peaks and can thus be regarded as the ultimate stereotactic and conformal radiation modalities $[6,8,9]$.

This is so not least since everywhere else outside the Bragg peak a rather low ionization density is obtained with easily repairable radiation damage by the HR and NHEJ processes. This implies that these three ions only induce severe cell inactivation in the tumor where all high doses Bragg peaks are deposited. Then only mild repair inducing and easily repaired low dose and ionization density cellular damage are deposited in surrounding normal tissues. This may therefore be called molecular radiation therapy since a high apoptotic and senescent response is exclusively induced in the tumor by simultaneously delivering a high dose and high ionization density. Since the normal tissues at the same time are exposed only to a low dose and low ionization density the optimal dose per fraction can be increased, and the number of fractions reduced from the classical 30 to about 10. Due to the fractionation window of normal tissue around 2 Gy per fraction at low ionization density [4] the optimal dose per fraction with these optimal light ion beams should still be around 2 Gy to normal tissue whereas the dose and biological effect to the tumor may be 2 to 3 times this value. Out of the approximately 75 DSB's produced at this dose level in normal tissue, around $99 \%$ of them are in average correctly repaired as discussed above [4,9]. The lightest ions beyond protons therefore have unique properties to cure tumors even in tissues that are critical

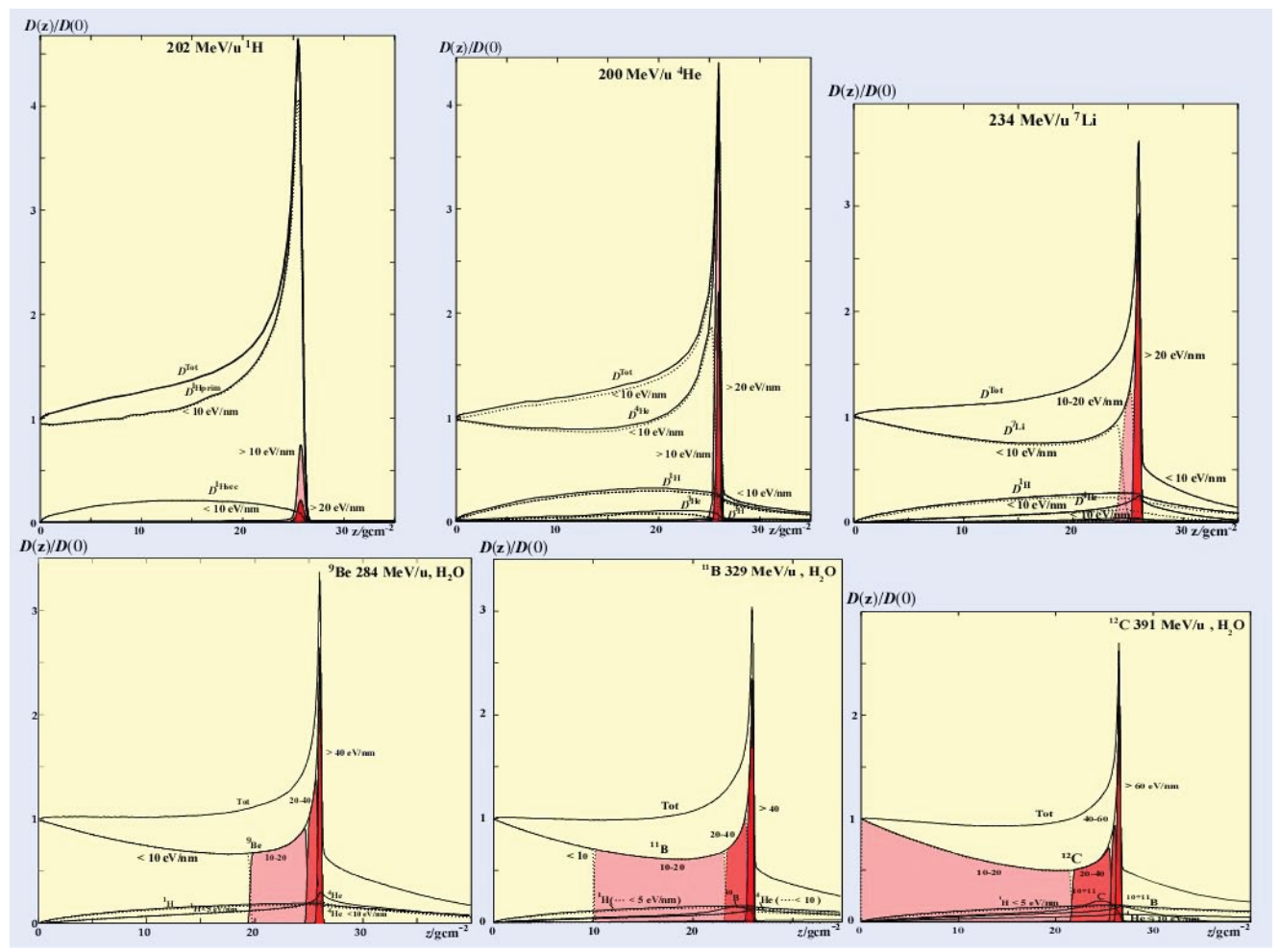

Figure 4: Depth dose curves for the 6 lightest ions: protons, Helium, Lithium, Beryllium, Boron and Carbon. The dose fraction delivered at an ionization density below $10 \mathrm{eV} / \mathrm{nm}$ is unshaded because when an ion passes the $2 \mathrm{~nm}$ DNA string less than $20 \mathrm{eV}$ are in average deposited so no ionization is generally obtained. It is clearly seen that Lithium has high ionization density only in the Bragg peak whereas Carbon has it about $4 \mathrm{~cm}$ to $5 \mathrm{~cm}$ in front and about $10 \mathrm{~cm}$ behind the Bragg peak. 


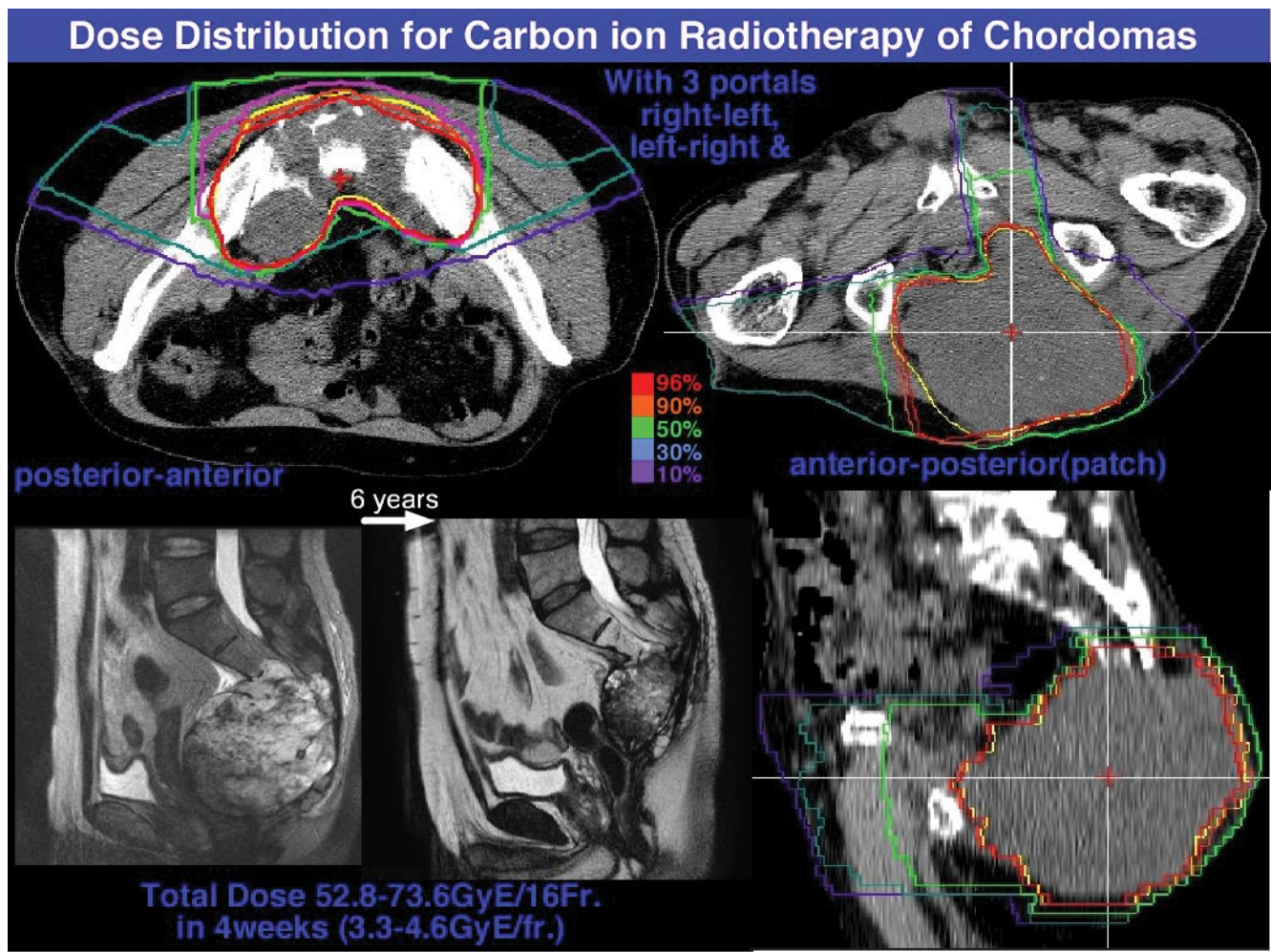

Figure 5: Illustration of the dose delivery for a large pelvic Chordoma shown in the upper and lower right treatment plans. The lower left plain CT images show the gradual disappearance of the chordoma six years after the treatment, probably due to a totally senescent response without traces of tumor growth (courtesy: H Tsujii, NIRS, Chiba).

for survival. A possible design of a very compact and cost efficient light ion installation is shown in Figure 6, illustrating the powerful advantage of a single eccentric gantry being able to treat as many as 3000 patients per year in four independent surrounding treatment rooms. The remaining challenge is to localize the tumor as accurate as possible in $3 \mathrm{D}$ in relation to anatomical reference points. A number of interesting developments of molecular tumor imaging are possible for this purpose as discussed below.

\section{High Resolution Ultra-Sensitive Whole Body Molecular PET Imaging}

PET and PET-CT have introduced a third revolution to tumor imaging after CT and MR. Their unique ability to allow highly specific molecular imaging with positron emitting tracer has improved tumor diagnostics. Unfortunately, the geometric resolution is only about $4 \mathrm{~mm}$ today in whole body cameras, whereas brain- and other small volume cameras may reach $1 \mathrm{~mm}$ and below already today. To get this kind of resolution, which is required for accurate radiation therapy, it is desirable to decrease the crystal size and increase the sensitivity by increasing the axial field of view from about $15 \mathrm{~cm}$ to $20 \mathrm{~cm}$ to about $100 \mathrm{~cm}$ to $120 \mathrm{~cm}$. This will increase the sensitivity about 50-fold and reduce the treatment time to a few minutes without needing to scan the patient or the camera to see the full extent of the disease (Figure 7). Therefore, even if the cost increases by a factor of five or so, it will be possible to substantially increase the patient throughput. Furthermore, it may become feasible to unfold the random positron diffusion to improve resolution owing to the much higher number of registered annihilation events. In addition, it will be important to remove respiration dynamics, so our idea is to combine the PET imaging in list mode with full 3D projection camera optical imaging so that each phase of the breathing cycle is fully synchronized with the PET dataset. It may then even be possible to project all the PET data to one fixed position of the breathing cycle, for example when simultaneous 4D CT data and projection camera data are available. With all these methods at work a fast whole body camera may reach a resolution of around $1 \mathrm{~mm}$.

The treatment could then be performed with breath hold in the phase where the complication free cure of the treatment is maximized or by synchronizing the treatment by a similar projection camera in the treatment room (Figure 8). In fact, it could be most cost efficient if all diagnostic and therapeutic units were provided with projection cameras for Auto Set Up and full breathing cycle synchronization.

This would allow almost perfect synchronization of all data sets whether using CT, MR or PET for dose delivery and biological responsiveness imaging (Figure 9). With advanced imaging equipment, it would generally be much less cost efficient if two or more very expensive units where totally integrated. Often they may not work simultaneously and one unit may be prohibited from use when the other is working. Furthermore, we may need more than two datasets for many situations. What one may save is the setup of the patient at two different units, but with projection camera Auto Set Up this is a minor problem and there are still motion artifacts between the two diagnostic modalities even if the initial positioning is correct.

The only other solution to all these simultaneous problems would be ultra-fast imaging and dose delivery by all methods requiring 
Citation: Brahme A (2016) High Resolution Molecular Radiation Therapy and Tumor Imaging for the $21^{\text {st }}$ Century. J Nucl Med Radiat Ther 7: 311 . doi: $10.4172 / 2155-9619.1000311$

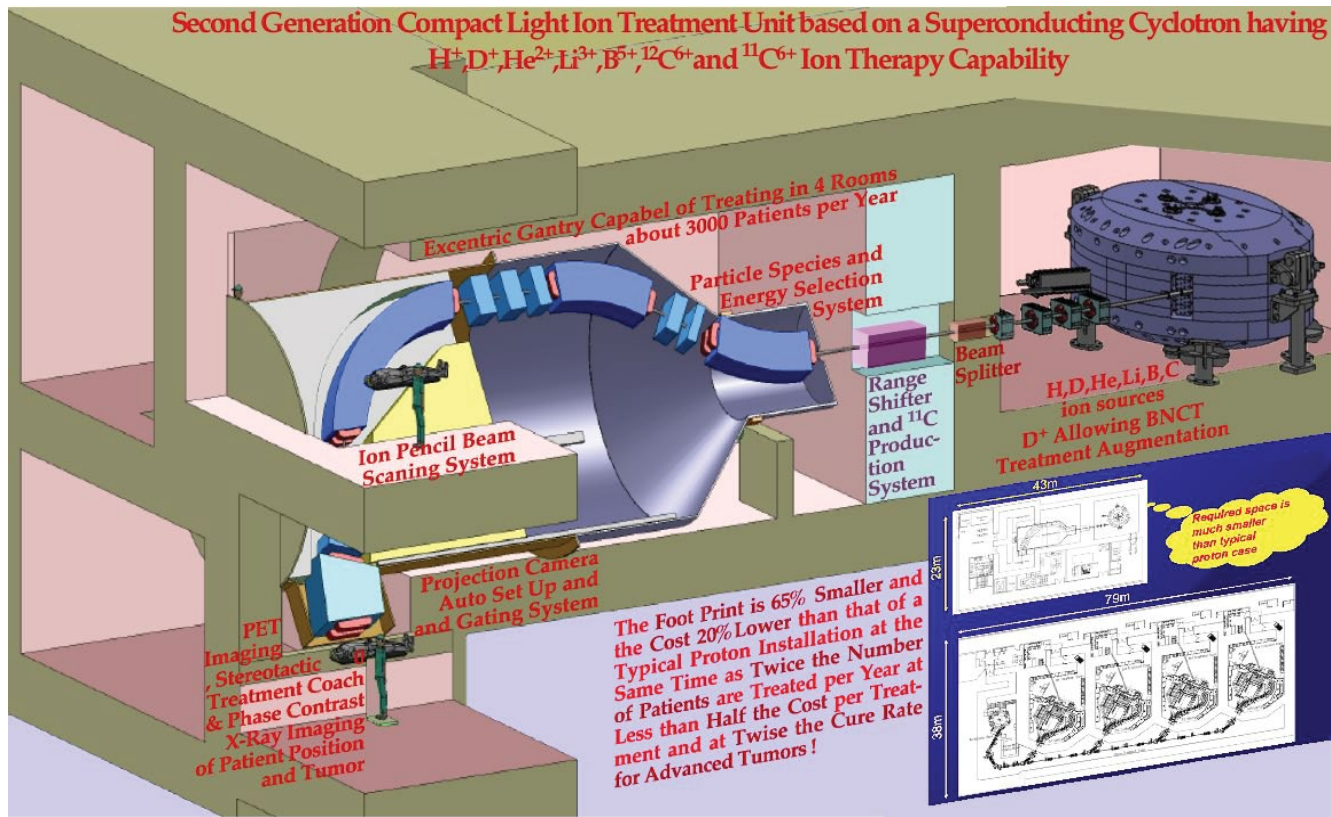

Figure 6: CAD-drawing of the superconducting cyclotron and the beam transport to the eccentric gantry and the four treatment rooms with the lower right one with a patient being set up for treatment. The performance of this very flexible second generation treatment unit is far beyond the traditional large synchrotron based installations at a fraction of the cost and footprint. The ability to use ${ }^{11} \mathrm{C}$ ions for treatment increases the Bragg peak specificity in dose delivery imaging about 50 -fold $[9,10]$

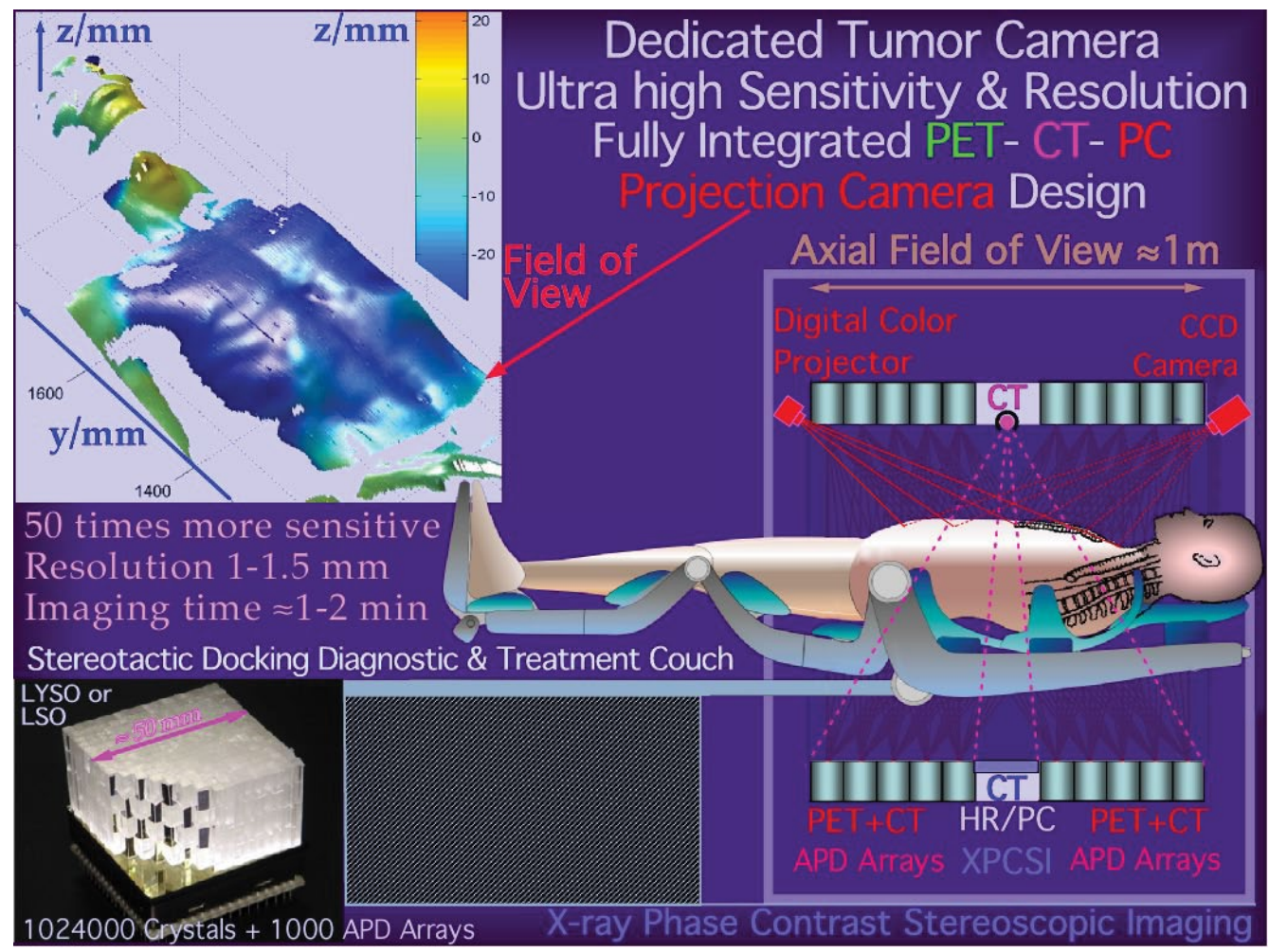

Figure 7: Cross section through a dedicated high-resolution open PET-CT tumor camera where the central opening is introduced to allow a high resolution in the central CT-region. The PET detectors may also detect peripheral CT photons but at a lower resolution sufficient for optimal PET reconstruction using CT attenuation data. The design may alternatively include high resolution stereoscopic Phase Contrast in the open PET configuration [11]. 
Citation: Brahme A (2016) High Resolution Molecular Radiation Therapy and Tumor Imaging for the $21^{\text {st }}$ Century. J Nucl Med Radiat Ther 7: 311 . doi: $10.4172 / 2155-9619.1000311$

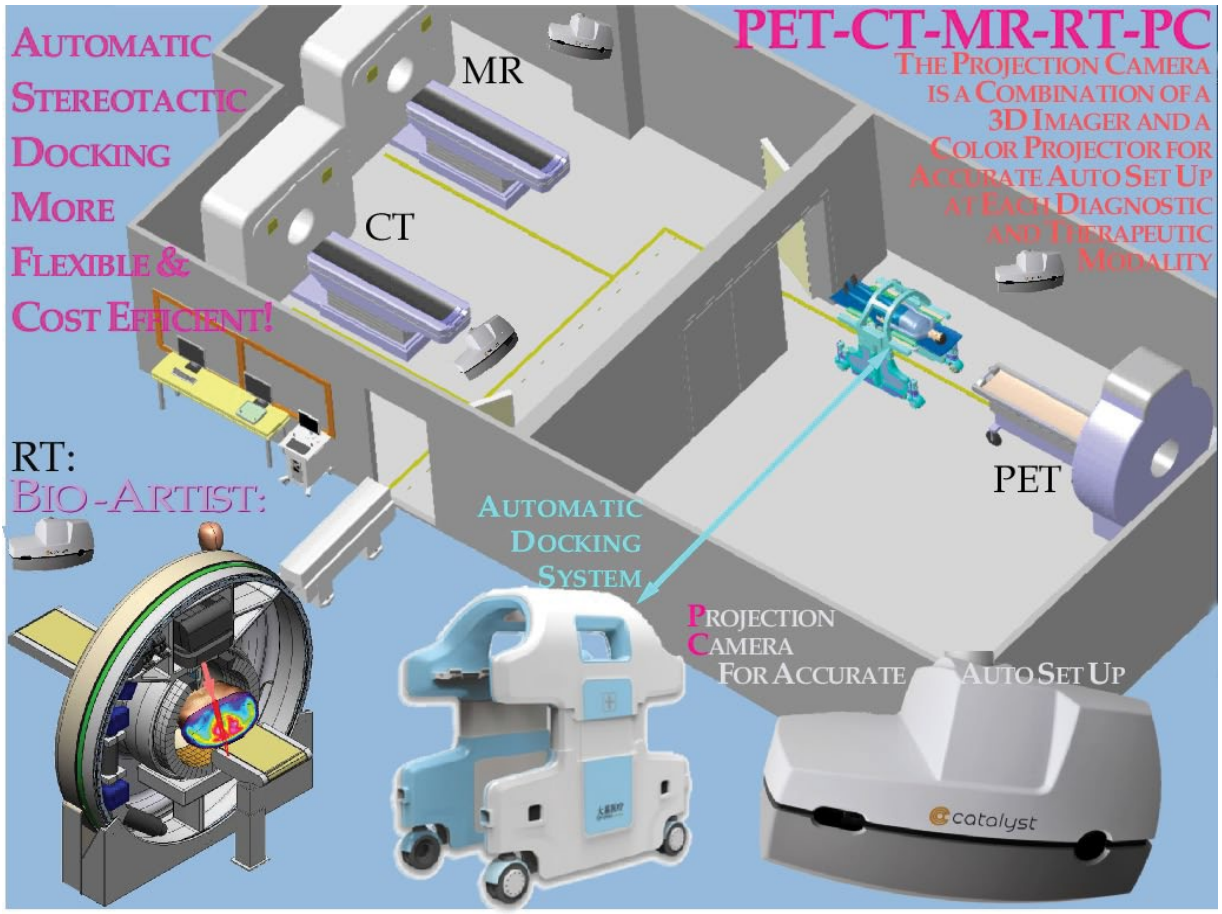

Figure 8: To improve cost effectiveness it is better to combine multiple diagnostic and therapeutic units all with accurate optical Auto Set Up units than to build together sub-optimal units so that they block each other's imaging ability. With an Auto Set Up robot all imaging and therapeutic units can be combined in any desired order with optimal performance of each unit making no compromises using MR-CT-PET-RT or lon Therapy for example.

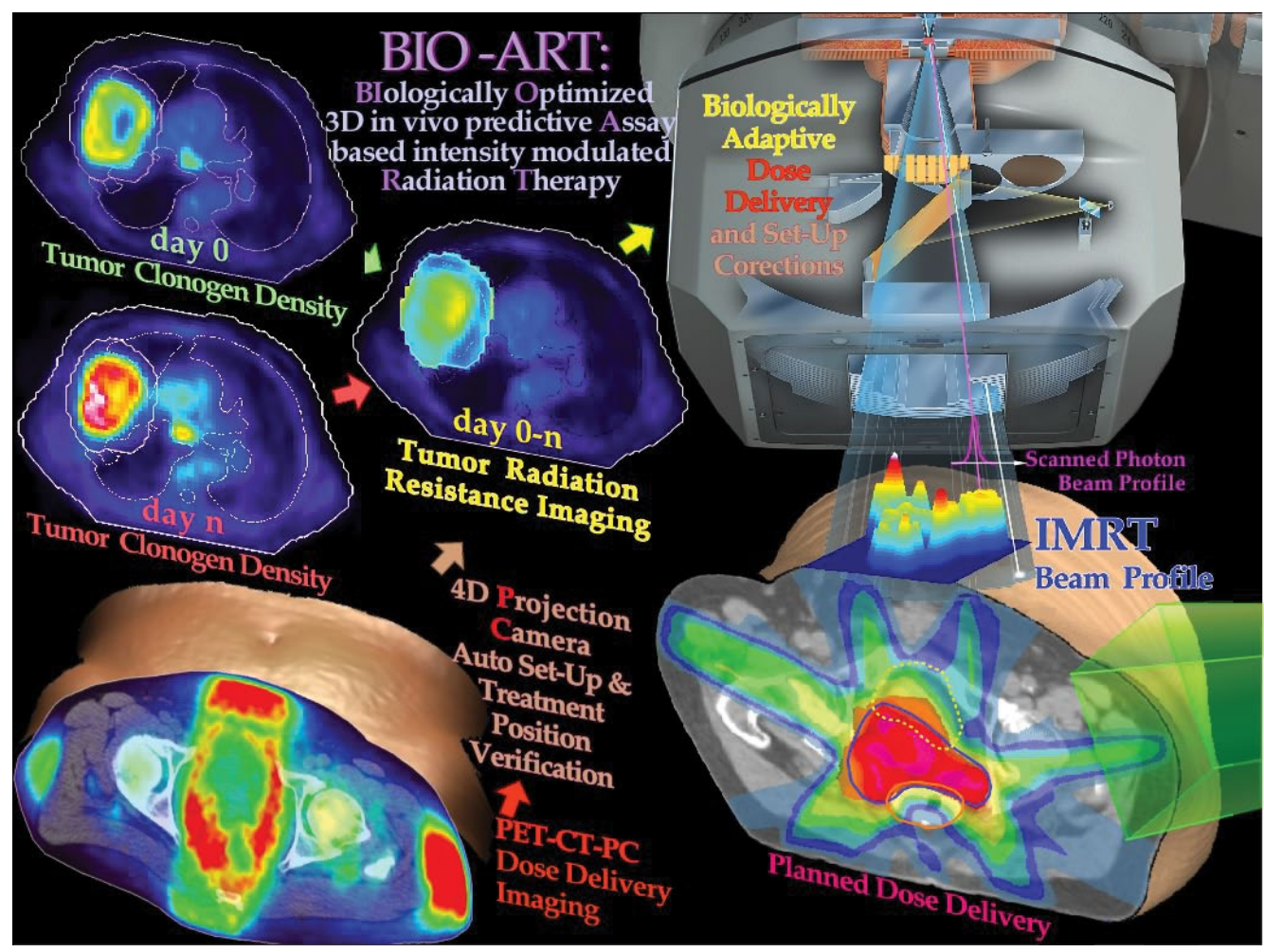

Figure 9: Illustration showing how PET-CT information can be used to plan, verify and biologically optimize radiation therapy using in vivo predictive assay (BIOART, [12]). By measuring the tumor cell kill early in the treatment, 3D in vivo predictive assay information about radiation responsiveness is obtained for accurate prediction of the optimal dose delivery based on a known hypoxic status. 
accurate synchronization of all diagnostic and therapeutic techniques. Interestingly, modern electronics allow the same detector to be used for CT and PET. Avalanche Photo diodes for example can be run in a low voltage current mode for CT and then be driven on a high voltage single photon counting mode for PET. Furthermore, the open PET design $[10,11]$ could be used to put a high contrast and resolution Phase Contrast CT, or even an ultrafast stereoscopic phase contrast X-ray unit in the middle of the PET camera where the PET sensitivity would also be maximal (Figure 7) $[12,13]$.

\section{High Resolution Tumor Metabolite Imaging by $15 \mathrm{~T}$ to 20 T Whole Body MRSI}

With conventional 3 Tesla MR units, the geometric resolution with tumor metabolite spectroscopic imaging is only around $10 \mathrm{~mm}$ to $15 \mathrm{~mm}$. This reduces the diagnostic imaging of true tumor tissue substantially, so large set-up margin may be needed, and a significant increase in the irradiation of normal tissues may result. At about 15 Tesla, the geometric resolution with tumor metabolite imaging may reach about $1 \mathrm{~mm}$ with substantial improvement in molecular spectral imaging for accurate radiation therapy planning.

The highest field strength whole body MR unit at present is the 12 Tesla INUMAC system, developed in collaboration between French and German scientists mainly for brain studies with $0 \mathrm{~mm}$ to $1 \mathrm{~mm}$ resolution (http://phys.org/news/2013-10-world-powerful-mri-online. $\mathrm{html}$ ). It should be possible to reduce the aperture somewhat from the present $900 \mathrm{~mm}$ of this unit and use the latest type of high field strength tolerating windings to get into the 15 Tesla to 20 Tesla region with sub $\mathrm{mm}$ spectral resolution. To further improve spectral resolution and reconstructing speed the scanner should use Fast Pade transform rather than Fourier transform as shown in Figure 10 [13]. This technique may further increase the effective magnetic field of the unit, which may be highly desirable for spectroscopic imaging. In Figure 11, spectral metabolite imaging of a high-resolution brain scanner is showing the substantial improvement in image resolution of a small bore camera and the value of using alternative metabolites for accurate target volume definition and confirmation [14].

\section{Ultra-High Resolution and Contrast Using Stereoscopic Phase Contrast X-Ray Imaging}

The CT technique has today reached sub $\mathrm{mm}$ resolution due to fast rotating X-ray units with 0 to $3 \mathrm{sec}$ revolution time. It will probably be quite hard to reach much faster rotational speeds to freeze fast organ motions in a live patient. Interestingly, Phase Contrast X-rays with their much higher contrast resolution may be a new way forward here. Due to the significantly increased contrast (Figure 12), it may even be possible to use 3D Stereoscopic imaging with just two projections, produced by two short pulsed X-ray flashes that may be as short as a couple of msec to $\mu \mathrm{sec}$, and thus effectively freeze all organ motions.

With a small effective local spot like the $40 \mu \mathrm{m}$ Tin-jet in Figure 12, a spatial resolution as well as $10 \mu \mathrm{m}$ may be achievable as shown in the figure. The increased phase contrast allows stereoscopic imaging with just two projections rather than the 400 or so needed with ordinary CT due to its low absorption contrast. In fact, with a small X-ray source the diffraction of the $\mathrm{X}$-rays will significantly improve image contrast and resolution as seen in the lower right insert, making 3D stereoscopic imaging feasible. A suitable set-up for fast stereoscopic imaging is
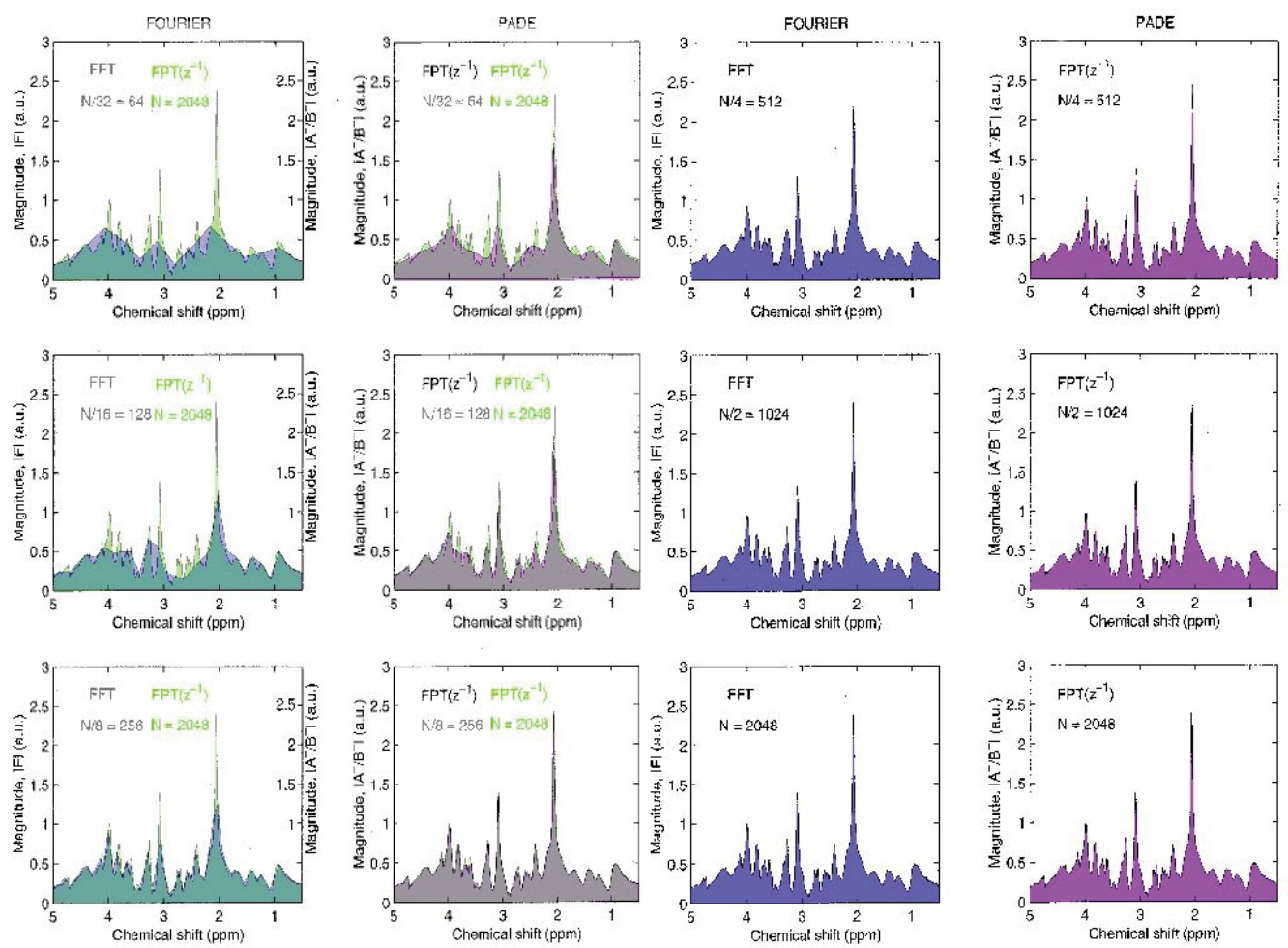

Figure 10: Illustration of the increased effectiveness of spectral reconstruction using the Pade transform compared to the commonly used FFT-method. Almost $1 / 3$ to $1 / 2$ as much iteration is sufficient with the Pade transform method (modified from [14]). 
shown in Figure 13, where the X-ray flash tubes and the high-resolution images are complemented by a projection camera to get fast additional 3D image information about the skin surface in analogy with the PET, MR, and radiation therapy set-up shown in Figure 8. The projection camera will help flashing the X-rays at the right phase of the breathing cycle without unnecessary X-ray exposer and with about $0 \mathrm{~mm}$ to $5 \mathrm{~mm}$ positional accuracy. As seen in Figure 14, this will significantly increase the spatial and temporal resolution of 3D X-ray imaging. Furthermore, if a quasi-monochromatic fluorescent X-ray tube is used with suitable energy for molecular specific tumor tracers, the specificity may reach a level otherwise achievable only by Synchrotrons or inverse Compton $\mathrm{X}$-ray sources [15].

Stereoscopic Phase Contrast X-rays may therefore become a $4^{\text {th }}$ molecular tumor imaging revolution, allowing cellular spatial resolutions and ultra-fast imaging time to freeze all organ motions at the same time as tumor specific molecular traces can be used to further increase contrast and specificity [15]. Cellular resolution and short imaging times may not always be needed with tumor imaging but instead a 10- to 100-fold reduction of X-ray exposer may be in reach. Interestingly, both PET-CT and MR-MRSI or CT may also increase both the spatial and contrast resolution as seen in Figure 14, but not as much as PCSXI can. However, the low dose and speed of PCSXI makes it an interesting complement also to PET imaging as indicated by the open PET-PCSXI camera in Figure 7. This will allow high resolution 3D X-ray imaging in the central $15 \mathrm{~cm}$ to $20 \mathrm{~cm}$ of the patient, often including the gross tumor volume at the same time as the PET sensitivity is about double in this region, even without any local positron detection [16-18].

\section{Conclusions}

The substantial potential improvements in diagnostic imaging will together with the new methods of Molecular Light Ion Therapy, significantly improve the early accurate diagnostics of tumors as well as their effective elimination by the mildest and most curative treatment modality available today, using nature's own preferred pathways for programmed cell death and senescence in the tumor (Figure 1). The high tumor specific resolution will help save normal tissues as far as possible at the same time as the tumor volume can be given high therapeutic doses. It is therefore essential that all diagnostic and therapeutic modalities have a resolution of around $1 \mathrm{~mm}$ to 0.5 $\mathrm{mm}$, which is possible with all modalities presently discussed. With all these diagnostic and therapeutic modalities in operation, maybe ten years from now, tumor cure probabilities of around $80 \%$ should be in reach [9]. Early detection by highly specific blood analysis has already reached $96 \%$ significance in detecting stage 1 pancreas cancer [19]. And the cancer progression and malignancy can even be estimated (e.g., by comparative genomic hybridization) [20]. With these early detection methods, the optimal treatment can be selected and even much higher cure rates will most likely be in reach. One may worry about the increased costs of advanced imaging and therapy in medical care. However, the simultaneously substantially improved performance reduces the clinical workload, saves time and improves treatment outcome so at the end often reduced costs may result, at least when applied in large cost effective departments where more regular equipment also exists to maximize cost effectiveness of the clinical care.

\section{Acknowledgements}

Continued fruitful discussions with Hirohiko Tsujii at the National Institute of Radiological Sciences are gratefully acknowledged.

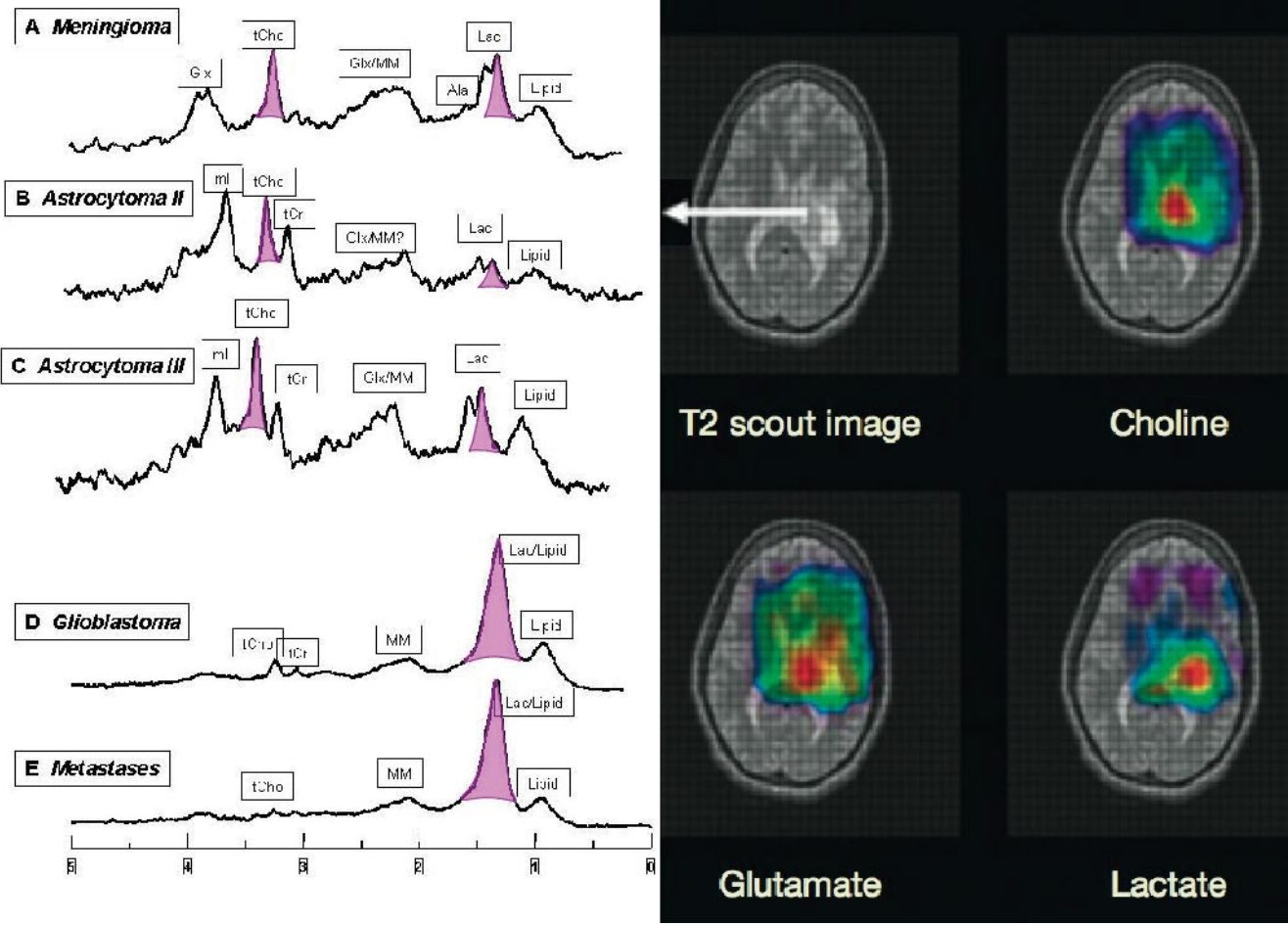

Figure 11: Brain MRS imaging with different metabolites of a brain tumor (modified from Zhou and Brahme [15] work). It is clearly seen that both choline and lactate give rather similar indication of the gross tumor localization. 


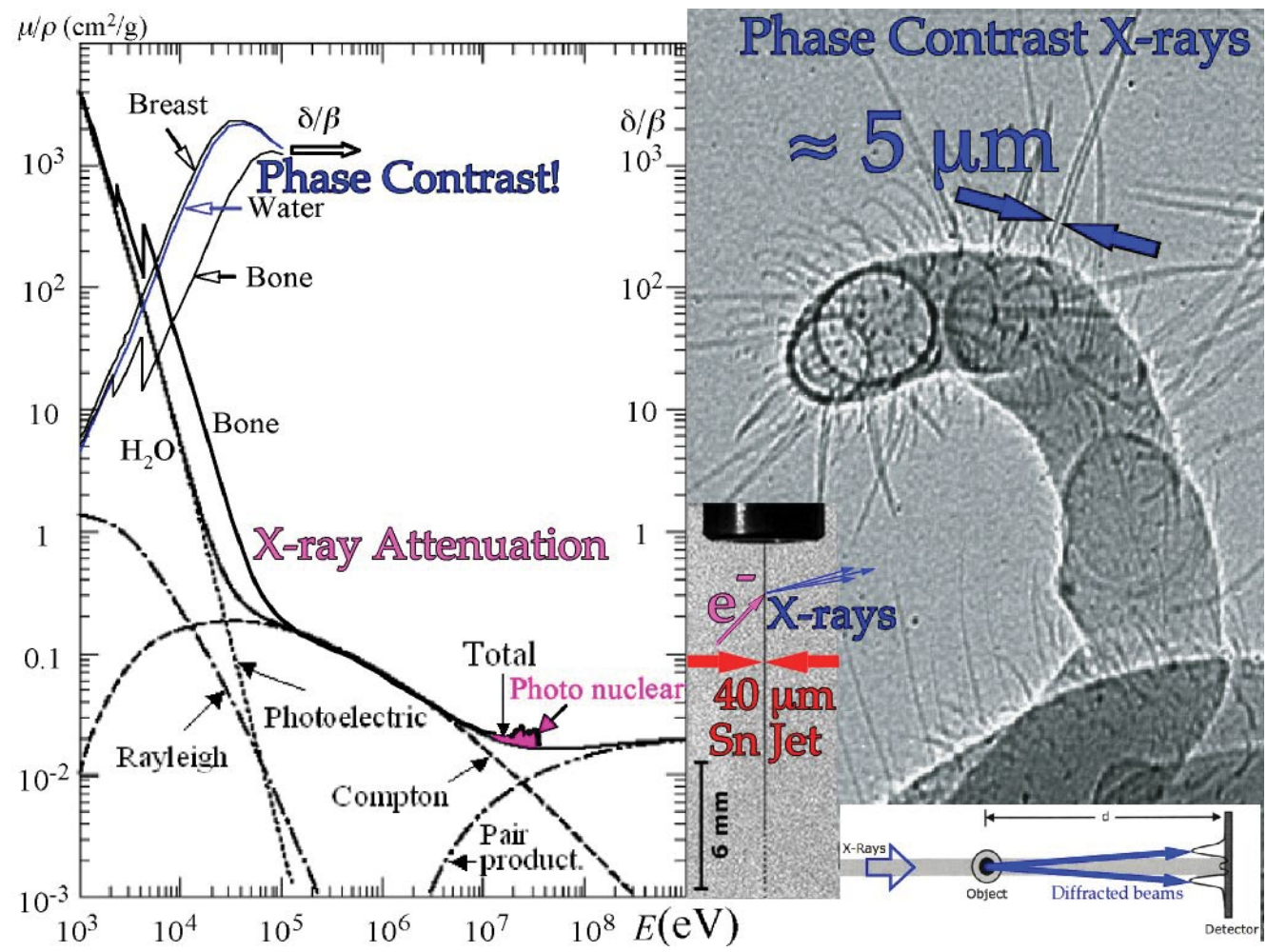

Figure 12: With Phase Contrast X-rays the contrast is increasing towards a few hundred keV rather than rapidly decreasing for ordinary X-ray absorption imaging This increased contrast and diffraction based edge X-ray imaging a potential method, replacing CT with a significantly reduced 3D imaging technique with two instead of some 400 projections. The increased contrast on different anatomical structures makes stereoscopic imaging a new fast and efficient 3D imaging method (modified from Yamaya et al. $[16,17])$

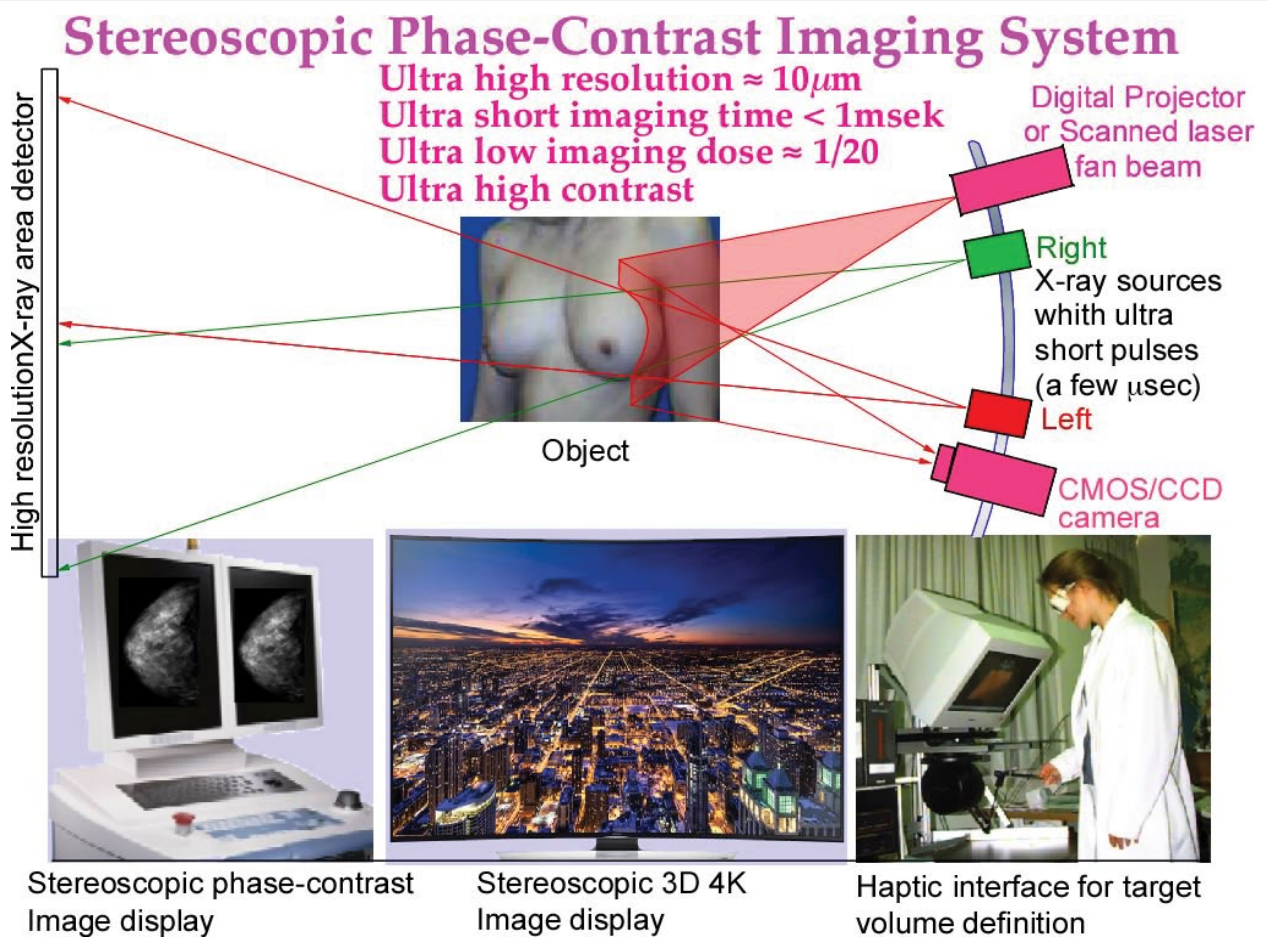

Figure 13: A possible design of a fast and cost efficient 3D phase contrast $X$-ray imaging method where an optical projection camera is used to help define the skin surface of the patient with sub $\mathrm{mm}$ accuracy. With two flash X-ray tubes the same imaging time is obtained for each projection. With a fast moving single X-ray tube imaging within $0.1 \mathrm{sec}$ should also be a possibility. However, to reach $5 \mu \mathrm{m}$ to $10 \mu \mathrm{m}$ whole body spatial resolution two X-ray tubes and simultaneous irradiation within a fraction of a millisecond or less will be needed. 


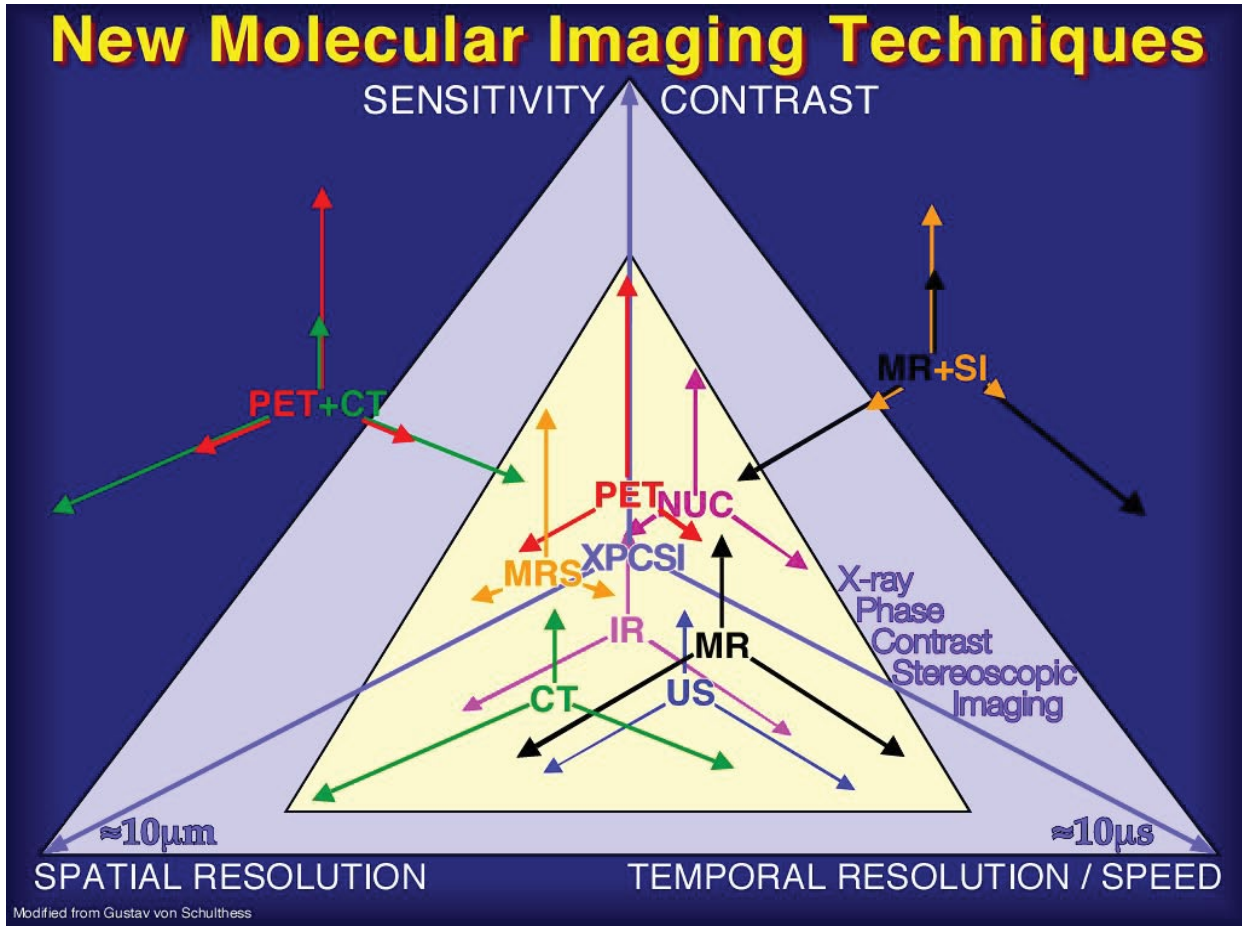

Figure 14: Tumor imaging for radiation therapy need a geometric resolution of about $1 \mathrm{~mm}$ or less to match the accuracy of dose delivery. Whole body MRSI and PET are not there yet and the present proposals may help achieving this. The most powerful 3D molecular imaging method is likely to become Stereoscopic Phase Contrast X-ray Imaging (SPCXI), with tenfold increase over ordinary CT and much more in speed using dual flash X-ray tubes as indicated in Figure 10.

\section{References}

1. Nakamura $Y(2004)$ Isolation of p53-target genes and their functional analysis. Cancer Sci 95: 7-11.

2. Brahme A (2007) Development of Highly Specific Molecular Cancer Therapy with the Lightest lons. $5^{\text {th }}$ Takahashi Memorial International Symposium, Book of Abstract 57, Japan.

3. Brahme A (2014) Biologically Optimized Light lon Therapy. In: Brahme A. Ed. in Chief, Comprehensive BioMedical Physics, Major Reference Work, Elsevier, Oxford, Amsterdam, Netherlands, pp: 529-554

4. Brahme A (2011) Accurate Description of the Cell Survival and Biological Effect at Low and High Doses and LETs. J Rad Res 52: 389-407.

5. Brahme A (2000) Development of radiation therapy optimization. Acta Onco 39: 579-595.

6. Brahme A (2010) Optimal use of light ions for radiation therapy. Hoshasen Kagaku 53: 35-61.

7. Vreede P, Brahme A (2009) Development of biologically optimized radiation therapy. Maximizing the apoptotic cell kill. Hoshasen Kagaku 52: 31-52.

8. Brahme A (2009) Potential developments of light ion therapy. The ultimate conformal treatment modality. Hoshasen Kagaku 52: 8-31.

9. Brahme A, Svensson H (2014) Physical, Biological and Clinical Background for the Development of Biologically Optimized Light lon Therapy. In: Brahme A, eds. Biologically Optimized Radiation Therapy. World Scientific Publishing, Singapore.

10. Brahme A (2010) Design of an Open PET System with Therapeutic \& Stereoscopic Phase-Contrast X-ray Capabilities. Proceedings of JapaneseEuropean Joint Symposium on lon Cancer Therapy and NIRS-KI Joint Symposium on Ion-Radiation Sciences, Stockholm.
11. Lazzeroni M, Brahme A (2015) Production of pure quasi-monochromatic $11 \mathrm{C}$ beams for accurate radiation therapy and dose delivery verification. Nucl Inst Meth Phys Res B 359: 120-130.

12. Brahme A (2014) BIOART: Biologically Optimized 3D InVivo Predictive Assay Based Radiation Therapy. In: Brahme A, eds. Biologically optimized radiation therapy. World Scientific Publishing, Singapore.

13. Belkic $Z$ (2001) Fast Padé Transform (FPT) for magnetic resonance imaging and computerized tomography. Nuc Inst Meth Phys Res A 471: 165-169.

14. Prost RW (2014) Magnetic Resonance Spectroscopic Imaging. In: Belkic D, Brahme A, eds. Comprehensive Biomedical Physics. Elsevier, Amsterdam, Netherlands, pp: 331-345.

15. Zhou SA, Brahme A (2010) Development of High-resolution molecular phasecontrast stereoscopic X-ray imaging for accurate cancer diagnostics. Rad Pro Dos 139: 334-338.

16. Yamaya T, Inaniwa T, Minohara S, Yoshida E, Inadama N, et al. (2008) A proposal of an open PET geometry. Phys Med Biol 53: 757-773.

17. Yamaya T, Inaniwa T, Minohara S, Yoshida E, Inadama N, et al. (2011) Development of a small prototype for a proof-of-concept of OpenPET imaging Phys Med Biol 56: 1123-1137.

18. Yamaya T, Yoshida E, Kinouchi S, Nakajima Y, Nishikido F, et al. (2012) A small prototype of a single-ring OpenPET. Nuclear Science Symposium and Medical Imaging Conference, IEEE, 27 Oct-3 Nov 2012, California, USA.

19. Gerdtsson SA, Wingren C, Persson H, Delfani P, Nordström M, et al. (2016) Plasma protein profiling in a stage defined pancreatic cancer cohort Implications for early diagnosis. Mol Oncol 10: 1305-1316.

20. Navin N, Krasnitz A, Rodgers L, Cook K, Meth J, et al. (2010) Inferring tumor progression from genomic heterogeneity. Genome Res 20: 68-80 